\title{
EXOTIC SOLUTIONS OF THE CONFORMAL SCALAR CURVATURE EQUATION IN $\mathbb{R}^{n}$
}

\author{
Man Chun LEUNG \\ Department of Mathematics, National University of Singapore, 2 Science Drive 2, \\ Singapore 117543, Singapore \\ Received 13 January 2000
}

ABSTRACT. - We construct global exotic solutions of the conformal scalar curvature equation $\Delta u+[n(n-2) / 4] K u^{(n+2) /(n-2)}=0$ in $\mathbb{R}^{n}$, with $K(x)$ approaching 1 near infinity in order as close to the critical exponent as possible.

(C) 2001 L'Association Publications de l'Institut Henri Poincaré. Published by Elsevier B.V. All rights reserved

Keywords: Conformal scalar curvature equation; Gluing solutions; Positive scalar curvature AMS classification: Primary 35J60; Secondary 58J05

RÉSUMÉ. - Nous construisons des solutions globales exotiques de l'équation courbure scalaire conforme $\Delta u+[n(n-2) / 4] K u^{(n+2) /(n-2)}=0$ dans $\mathbb{R}^{n}$, avec $K(x) \rightarrow 1$ quand $|x| \rightarrow \infty$ (C) 2001 L'Association Publications de l'Institut Henri Poincaré. Published by Elsevier B.V. All rights reserved

\section{Introduction}

We consider a special class of positive solutions of the conformal scalar curvature equation

$$
\Delta u+\frac{n(n-2)}{4} K u^{\frac{n+2}{n-2}}=0 \quad \text { in } \mathbb{R}^{n} .
$$

Here $\Delta$ is the standard Laplacian on $\mathbb{R}^{n}$ equipped with Euclidean metric $g_{o}, K$ a smooth function on $\mathbb{R}^{n}$, and $n \geqslant 3$ an integer. The solutions we construct breach a rather natural lower bound and have peculiar asymptotic property.

Eq. (1.1) is studied extensively by many authors in connection with the prescribed scalar curvature problem on a Riemannian manifold in general and on $\mathbb{R}^{n}$ and $S^{n}$ in particular (on $S^{2}$, the Nirenberg problem; cf. $[1,3-5,9,12,14,15,17,20,21,23,24,26]$ and the references within). As in the case of the Yamabe problem, recent studies indicate that the case when $K$ is strictly positive affords many interesting and subtle developments.

Assume that $K$ is bounded between two positive constants in $\mathbb{R}^{n}$. An important feature of Eq. (1.1) is the asymptotic behavior of $u(x)$ for large $|x|$ (cf. [2,5-8,10,12,16,18,19,

E-mail address: matlmc@math.nus.edu.sg (M.C. Leung). 
22]). It is simpler to classify with the help of the Kelvin transformation:

$$
y=\frac{x}{|x|^{2}} \quad \text { and } \quad w(y):=|y|^{2-n} u\left(y /|y|^{2}\right) \quad \text { for } x, y \in \mathbb{R}^{n} \backslash\{0\} .
$$

From (1.2), $w$ satisfies the equation

$$
\Delta w(y)+\frac{n(n-2)}{4} \bar{K}(y) w^{\frac{n+2}{n-2}}(y)=0 \quad \text { for } y \in \mathbb{R}^{n} \backslash\{0\},
$$

where $\bar{K}(y):=K\left(y /|y|^{2}\right)$ for $y \neq 0$ (see, for instance, [18]). $w$ (and $u$ ) is said to have fast decay if $w$ has a removable singularity at the origin. Otherwise, it is called a singular solution. In order to have reasonable control on the geometric and analytic behavior of singular solutions, it is crucial to obtain the upper bound or slow decay

$$
w(y) \leqslant C_{1}|y|^{-(n-2) / 2} \quad \text { as } y \rightarrow 0, \quad \text { i.e., } \quad u(x) \leqslant C_{1}|x|^{-(n-2) / 2} \quad \text { for }|x| \gg 1,
$$

where $C_{1}$ is a positive constant. The question on slow decay is discussed in depth in [2, $5-8,16,18,19,22]$ (cf. also [27]; note that our definition of slow decay is slightly different from the one in [5] and [8]). Guided by the case when $K$ is equal to a positive constant outside a compact subset of $\mathbb{R}^{n}$ (see $[2,16]$ ), it is natural to ask whether a singular positive solution $u$ with slow decay also satisfies the lower bound

$$
w(y) \geqslant C_{2}|y|^{-(n-2) / 2} \quad \text { as } y \rightarrow 0, \quad \text { i.e., } \quad u(x) \geqslant C_{2}|x|^{-(n-2) / 2} \quad \text { for }|x| \gg 1,
$$

where $C_{2}$ is a positive constant. If the lower bound holds, then the conformal metric $u^{4 /(n-2)} g_{o}$ on $\mathbb{R}^{n}$ is complete and has bounded (sectional) curvature [8]. The radial Pohozaev number is an essential invariant in the study of equation (1.1) and is given by

$$
P(u):=\lim _{R \rightarrow \infty} \int_{B_{o}(R)}[x \cdot \nabla K(x)] u^{2 n /(n-2)}(x) \mathrm{d} x,
$$

provided the limit exists. Here $B_{o}(R)$ is the open ball with center at the origin and radius equal to $R>0$. The following result is shown by Chen and Lin in [6] and [8], mindful of the slightly different notations we use.

THEOREM 1.7 (Chen-Lin). - Let u be a positive smooth solution of Eq. (1.1). Assume that $\lim _{|x| \rightarrow \infty} K(x)$ exists and is positive, and there exist positive constants $l \geqslant(n-2) / 2$ and $C$ such that

$$
C^{-1}|x|^{-(l+1)} \leqslant|\nabla K(x)| \leqslant C|x|^{-(l+1)} \text { for all }|x| \gg 1 .
$$

Then $u$ has slow decay and $P(u)$ exists and is non-positive. $u$ has fast decay if and only if $P(u)=0$ (the Kazdan-Warner condition). Furthermore, if $u$ is a singular solution, then we also have the lower bound $u(x) \geqslant C_{2}|x|^{-(n-2) / 2}$ for all $|x| \gg 1$ and for some positive constant $C_{2}$. 
More generally, under the condition that $\lim _{|x| \rightarrow \infty} K(x)$ exists and is positive, and $|\nabla K|$ is bounded in $\mathbb{R}^{n}$, for a positive smooth solution $u$ of Eq. (1.1) with slow decay, we show in [10] (cf. also [5,8]) that $P(u) \leqslant 0$ if $P(u)$ exists. Moreover, $P(u)=0$ if and only if

$$
\liminf _{|x| \rightarrow \infty}|x|^{(n-2) / 2} u(x)=0 .
$$

In the latter case, the assumption on $K$ is not strong enough to allow us to deduce that $u$ has fast decay.

DEFINITION 1.9. - We call a singular positive solution $u$ of Eq. (1.1) with slow decay an exotic solution if (1.8) holds for $u$. That is, we cannot find a positive constant $C_{2}$ such that $u(x) \geqslant C_{2}|x|^{-(n-2) / 2}$ for all $|x| \gg 1$.

Then it is necessary that $P(u)=0$ if $P(u)$ exists. Exotic solutions are rather peculiar because from $P(u)=0$ one would expect $u$ to have fast decay. Instead, they decay slowly and the conformal metric $u^{4 /(n-2)} g_{o}$ remains to be complete, but the (sectional) curvature is unbounded [8]. Theorem 1.7 leads to the observation that there are no exotic solutions if $|\nabla K|$ decays to zero near infinity fast enough.

(Local) Exotic solutions are first found by Chen and Lin in [8]. By a scaling and the Kelvin transform, we may consider the equation

$$
\Delta u+\bar{K} u^{\frac{n+2}{n-2}}=0 \quad \text { in } B_{o}(1) \backslash\{0\} .
$$

Assume that $\bar{K}$ is radial and non-increasing in $(0,1]$, and is given by

$$
\bar{K}(r)=1-A r^{l}+R(r)
$$

for $r>0$ close to zero. Here $A>0$ and $0<l<(n-2) / 2$ are constants, and $R(r)=\mathrm{o}\left(r^{l}\right)$ and $R^{\prime}(r)=\mathrm{o}\left(r^{l-1}\right)$ for $r>0$ close to zero. Given a positive number $\alpha$, let $u(r, \alpha)$ be the unique solution of the initial value problem

$$
\left\{\begin{array}{l}
u^{\prime \prime}(r)+\frac{n-1}{r} u^{\prime}(r)+\bar{K}(r) u^{\frac{n+2}{n-2}}(r)=0 \\
u(0)=\alpha \text { and } u^{\prime}(0)=0
\end{array}\right.
$$

Chen and Lin [8] show elegantly that there exists a sequence $\alpha_{i} \rightarrow \infty$ such that $u\left(r, \alpha_{i}\right)$ converges to an (local) exotic $C^{2}$-solution of Eq. (1.10) in $B_{o}(1) \backslash\{0\}$. Subsequently, Lin [22] obtains characterizations of exotic solutions in terms of the asymptotic expansion of $\bar{K}$ near the origin.

The exponent $(n-2) / 2$ is found to be critical. For $l \geqslant(n-2) / 2$, Theorem 1.7 shows that there are no exotic solutions of Eq. (1.1). In this paper we construct global exotic solutions of Eq. (1.1) in $\mathbb{R}^{n}$. As described above, in [8], an abstract existence argument is used to show the existence of (local) exotic solutions. Our construction is explicit by gluing the Delaunay-Fowler-type solutions. Given any positive number $\delta$, we show that there is an exotic solution of Eq. (1.1) with $|K-1| \leqslant \delta^{2}$ in $\mathbb{R}^{n}$. Moreover, with regard to the critical exponent $(n-2) / 2$, we show that, given any positive function $\varphi(r)$ defined 
for $r \gg 1$ such that

$$
r^{(n-2) / 2} \varphi(r) \text { is non-decreasing for } r \gg 1 \text { and } \lim _{r \rightarrow \infty} r^{(n-2) / 2} \varphi(r)=\infty,
$$

(for example, $\varphi(r)=r^{-(n-2) / 2} \ln (\ln r)$ for $r \gg 1$ ), we construct an exotic solution of Eq. (1.1) with

$$
|K(x)-1| \leqslant C_{3} \varphi(|x|) \text { for all }|x| \gg 1,
$$

where $C_{3}$ is a positive constant. The analytic property of exotic solutions resides in a neighborhood of infinity, or, by the Kelvin transformation, on a neighborhood of the origin. Our emphasis on the whole $\mathbb{R}^{n}$ reflects the geometric viewpoint of conformal deformations of Euclidean space $\left(\mathbb{R}^{n}, g_{o}\right)$. We follow the convention of using $c, C$, $C^{\prime}, C_{1}, \ldots$ to denote positive constants, whose actual values may differ from section to section.

\section{Delaunay-Fowler-type solutions}

Introduce polar coordinates $(r, \theta)$ in $\mathbb{R}^{n}$, where $r=|x|$ and $\theta=x /|x|$ for $x \in \mathbb{R}^{n} \backslash\{0\}$. Let $t=\ln r$ for $r>0$ and

$$
v(t, \theta)=r^{(n-2) / 2} u(r, \theta) \quad \text { for } r>0 \text { and } \theta \in S^{n-1} .
$$

By the above transformation, Eq. (1.1) can be re-written as

$$
\frac{\partial^{2} v}{\partial t^{2}}+\Delta_{\theta} v-\frac{(n-2)^{2}}{4} v+\frac{n(n-2)}{4} \tilde{K} v^{\frac{n+2}{n-2}}=0 \quad \text { in } \mathbb{R} \times S^{n-1} .
$$

Here $\Delta_{\theta}$ is the Laplacian on the standard unit sphere in $\mathbb{R}^{n}$ and $\tilde{K}(t, \theta):=K(x)$, where $|x|=\mathrm{e}^{t}$ and $x /|x|=\theta$. For the case $\tilde{K} \equiv 1$ in $\mathbb{R} \times S^{n-1}$, consider radial solutions $v$ of (2.2) and the ODE

$$
v^{\prime \prime}-\frac{(n-2)^{2}}{4} v+\frac{n(n-2)}{4} v^{\frac{n+2}{n-2}}=0 \quad \text { in } \mathbb{R} .
$$

In connection with the study of surfaces of revolution of constant curvature by Delaunay [11] and a class of semilinear differential equations by Fowler [13], positive smooth solutions of Eq. (2.3) are known as Delaunay-Fowler-type solutions. We refer to [16,24, 25] for basic properties of the solutions. Eq. (2.3) is autonomous and the Hamiltonian energy

$$
H\left(v, v^{\prime}\right)=\left(v^{\prime}\right)^{2}-\frac{(n-2)^{2}}{4}\left[v^{2}-v^{2 n /(n-2)}\right]
$$

is constant along solutions of (2.3). For a positive smooth solution $v$ of (2.3), $H$ is a nonpositive constant in the interval $\left[-[(n-2) / n]^{n / 2}(n-2) / 2,0\right]$ (see [16]). By shifting the parameter, we may normalize the solution so that

$$
v(0)=\max _{t \in \mathbb{R}} v(t) .
$$


Let $v_{o}$ be a positive solution of Eq. (2.3) with $H=0$. Under the normalization, we have

$$
v_{o}(t)=(\cosh t)^{(2-n) / 2} \quad \text { for } t \in \mathbb{R} .
$$

We note that, by the transformation in (2.1), $v_{o}$ corresponds to

$$
u_{o}(x)=\left(\frac{2}{1+|x|^{2}}\right)^{(n-2) / 2} \text { for } x \in \mathbb{R}^{n},
$$

which is a solution of Eq. (1.1) when $K \equiv 1$ in $\mathbb{R}^{n}$. In particular, $u_{o}$ is smooth near 0 , which corresponds to $s \rightarrow-\infty$ for $v_{o}$. The other extreme is when $H=-[(n-$ $2) / n]^{n / 2}(n-2) / 2$, and the corresponding solution $v$ is a constant function given by $v(t)=[(n-2) / n]^{(n-2) / 4}$ for $t \in \mathbb{R}$.

For $H \in\left(-[(n-2) / n]^{n / 2}(n-2) / 2,0\right)$, the solution can be indexed by the parameter $\varepsilon=\min _{t \in \mathbb{R}} v(t)$, which is called the neck-size of the solution, or the Fowler parameter. We have $\varepsilon \in\left(0,[(n-2) / n]^{(n-2) / 4}\right)$ and

$$
H=H(\varepsilon)=\frac{(n-2)^{2}}{4}\left[\varepsilon^{2 n /(n-2)}-\varepsilon^{2}\right] .
$$

Denote the normalized positive solution by $v_{\varepsilon}$, where $0<\varepsilon<[(n-2) / n]^{(n-2) / 4}$. It is known that $v_{\varepsilon}$ is periodic with period $T_{\varepsilon}$. Moreover, we always have [16]

$$
\varepsilon \leqslant v_{\varepsilon}(t) \leqslant v_{\varepsilon}(0)<1 \quad \text { for } t \in \mathbb{R} .
$$

The following result is essentially proved in [24] (cf. also [16]).

LEMMA 2.10. $T_{\varepsilon}$, the period of $v_{\varepsilon}$, is monotone in $\varepsilon$ for $\varepsilon \in\left(0,[(n-2) / n]^{(n-2) / 4}\right)$. We have $T_{\varepsilon} \rightarrow 2 \pi / \sqrt{n-2}$ as $\varepsilon \rightarrow[(n-2) / n]^{(n-2) / 4}$ and $T_{\varepsilon} \rightarrow \infty$ as $\varepsilon \rightarrow 0^{+}$. Furthermore, there exists a positive constant $C$, independent on $\varepsilon$, such that

$$
-\frac{4}{n-2} \ln (C \varepsilon) \leqslant T_{\varepsilon} \leqslant-\frac{4}{n-2} \ln \left(C^{-1} \varepsilon\right) \quad \text { as } \varepsilon \rightarrow 0^{+} .
$$

It is also known that $v_{\varepsilon}$ converges uniformly in compact subsets of $\mathbb{R}$ to the constant solution as $\varepsilon \rightarrow[(n-2) / n]^{(n-2) / 4}$, and to $v_{o}(t)=(\cosh t)^{(2-n) / 2}$ as $\varepsilon \rightarrow 0^{+}[16]$. For applications in Section 3 , we study the order of the latter convergence in more detail. As $H$ is constant along solutions, we have

$$
H\left(v_{\varepsilon}, v_{\varepsilon}^{\prime}\right)=-\frac{(n-2)^{2}}{4}\left(\varepsilon^{2}-\varepsilon^{2 n /(n-2)}\right)=-\frac{(n-2)^{2}}{4}\left[v_{\varepsilon}^{2}(0)-v_{\varepsilon}^{2 n /(n-2)}(0)\right]
$$

for $\varepsilon \in\left(0,[(n-2) / n]^{(n-2) / 4}\right)$. Thus we obtain

$$
v_{\varepsilon}^{2}(0)\left[1-v_{\varepsilon}^{4 /(n-2)}(0)\right]=\varepsilon^{2}\left(1-\varepsilon^{4 /(n-2)}\right)=-\frac{4 H}{(n-2)^{2}} .
$$

As $v_{\varepsilon}(0)>\varepsilon$ when $\varepsilon \rightarrow 0^{+}$, it follows from (2.12) that $v_{\varepsilon}(0) \rightarrow 1$ and $\varepsilon \rightarrow 0^{+}$. Furthermore,

$$
1-v_{\varepsilon}^{4 /(n-2)}(0)=\mathrm{O}\left(\varepsilon^{2}\right)
$$


We have

$$
v_{\varepsilon}(0)=\left[1+\mathrm{O}\left(\varepsilon^{2}\right)\right]^{(n-2) / 4}=1+\mathrm{O}\left(\varepsilon^{2}\right) \quad \text { as } \varepsilon \rightarrow 0^{+} .
$$

Hence there exists a positive constant $C_{n}$ which depends on $n$ only, such that

$$
\left|v_{\varepsilon}(0)-1\right| \leqslant C_{n} \varepsilon^{2} \quad \text { for } \varepsilon>0 \text { small. }
$$

We use the following well-known inequalities a number of times; they can be derived by simple integration methods. For positive constants $c$ and $\alpha \geqslant 1$, we have

$$
\left|x^{\alpha}-y^{\alpha}\right| \leqslant C|x-y| \text { for } 0 \leqslant x, y \leqslant c,
$$

where $C=C(\alpha, c)$ is a positive constant; moreover, for $\beta>0$,

$$
(1+z)^{\beta}=1+\mathrm{O}(|z|) \quad \text { as } z \rightarrow 0 .
$$

With $v_{o}$ given by (2.6), it follows from (2.9) and (2.15) that

$$
\left|v_{\varepsilon}^{\frac{n+2}{n-2}}(t)-v_{o}^{\frac{n+2}{n-2}}(t)\right| \leqslant c_{n}\left|v_{\varepsilon}(t)-v_{o}(t)\right|,
$$

where $c_{n}$ is a positive constant depending on $n$ only. Using Eq. (2.3) we have

$$
\begin{aligned}
\left|v_{\varepsilon}^{\prime \prime}(t)-v_{o}^{\prime \prime}(t)\right| & \leqslant \frac{(n-2)^{2}}{4}\left|v_{\varepsilon}(t)-v_{o}(t)\right|+\frac{n(n-2)}{4}\left|v_{\varepsilon}^{\frac{n+2}{n-2}}(t)-v_{o}^{\frac{n+2}{n-2}}(t)\right| \\
& \leqslant\left[\frac{(n-2)^{2}}{4}+\frac{n(n-2)}{4} c_{n}\right]\left|v_{\varepsilon}(t)-v_{o}(t)\right| \\
& =\bar{C}_{n}\left|v_{\varepsilon}(t)-v_{o}(t)\right|,
\end{aligned}
$$

where $\bar{C}_{n}$ is the positive constant defined in the formula. We claim that

$$
\left|v_{\varepsilon}^{\prime \prime}(t)-v_{o}^{\prime \prime}(t)\right| \leqslant 2 C_{n} \bar{C}_{n} \varepsilon^{2} \quad \text { for } t \in[0, \rho],
$$

where $\rho:=1 /\left(2 C_{n} \bar{C}_{n}\right)$. Here $C_{n}$ and $C_{n}^{\prime}$ are the positive constants in (2.14) and (2.18), respectively. Without loss of generality, we may assume that $\rho<C_{n}$. By (2.14) and (2.18), the bound holds on a neighborhood of 0 . Suppose that it holds on $[0, \sigma]$ for some positive number $\sigma$ less than $\rho$. As $v_{\varepsilon}^{\prime}(0)=v_{o}^{\prime}(0)=0$, we have

$$
\left|v_{\varepsilon}^{\prime}(t)-v_{o}^{\prime}(t)\right| \leqslant 2 C_{n} \bar{C}_{n} \varepsilon^{2} \sigma \leqslant \varepsilon^{2} \quad \text { for } t \in[0, \sigma] .
$$

Hence

$$
\left|v_{\varepsilon}(t)-v_{o}(t)\right| \leqslant\left(C_{n}+\sigma\right) \varepsilon^{2}<2 C_{n} \varepsilon^{2} \quad \text { for } t \in[0, \sigma] .
$$

By (2.18) we have

$$
\left|v_{\varepsilon}^{\prime \prime}(\sigma)-v_{o}^{\prime \prime}(\sigma)\right|<2 C_{n} \bar{C}_{n} \varepsilon^{2} .
$$

Using an connectedness argument, we obtain (2.19) as claimed. A similar bound holds in $[-\rho, 0]$. Upon integration we obtain the following lemma. 
LEMMA 2.21. - Let $v_{\varepsilon}$ and $v_{o}$ be the solutions of Eq. (2.3) discussed above. There exists positive constants $\rho$ and $C_{o}$ which depend on $n$ but not on (small enough positive) $\varepsilon$, such that

$$
\left|v_{\varepsilon}(t)-v_{o}(t)\right| \leqslant C_{o} \varepsilon^{2}, \quad\left|v_{\varepsilon}^{\prime}(t)-v_{o}^{\prime}(t)\right| \leqslant C_{o} \varepsilon^{2} \quad \text { and } \quad v_{\varepsilon}(t) \geqslant 1 / 2
$$

for $t \in[-\rho, \rho]$ and $\varepsilon>0$ close to 0 .

\section{Gluing solutions}

We follow the notations used in Section 2 and consider (2.1) and Eq. (2.2). Slow decay for a positive smooth solution $u$ of equation (1.1) corresponds to $v(s, \theta) \leqslant C$ for $s \gg 1, \theta \in S^{n-1}$ and a positive constant $C$. Moreover, $u$ is an (global) exotic solution if and only if there exists a sequence $\left\{\left(s_{i}, \theta_{i}\right)\right\} \subset \mathbb{R} \times S^{n-1}$ such that $\lim _{i \rightarrow \infty} s_{i}=\infty$ and $\lim _{i \rightarrow \infty} v\left(s_{i}, \theta_{i}\right)=0$, and, when the variable $t$ is changed into $r$ via $t=\ln r, u$ is smooth across the origin. Let $\phi_{1}$ be a smooth function on $\mathbb{R}$ such that $0 \leqslant \phi \leqslant 1$ in $\mathbb{R}$ and

$$
\phi_{1}(t)= \begin{cases}1 & \text { for } t \leqslant-\rho \\ 0 & \text { for } t \geqslant \rho\end{cases}
$$

We also require that

$$
\left|\phi_{1}^{\prime}(t)\right| \leqslant 2 / \rho \quad \text { and } \quad\left|\phi_{1}^{\prime \prime}(t)\right| \leqslant 2 / \rho^{2} \quad \text { for } t \in(-\rho, \rho) .
$$

Let $\phi_{2}=1-\phi_{1}$ in $\mathbb{R}$. Define

$$
v=\phi_{1} v_{o}+\phi_{2} v_{\varepsilon} \quad \text { in } \mathbb{R},
$$

where $\varepsilon>0$ is close to zero. It follows that

$$
\begin{aligned}
-v^{\prime \prime}(t)+\frac{(n-2)^{2}}{4} v(t)= & \frac{n(n-2)}{4}\left[\phi_{1} v_{o}^{\frac{n+2}{n-2}}(t)+\phi_{2} v_{\varepsilon}^{\frac{n+2}{n-2}}(t)\right]+\phi_{1}^{\prime}(t)\left[v_{\varepsilon}^{\prime}(t)-v_{o}^{\prime}(t)\right] \\
& +\phi_{1}^{\prime \prime}(t)\left[v_{\varepsilon}(t)-v_{o}(t)\right]
\end{aligned}
$$

for $t \in \mathbb{R}$. We also have

$$
\begin{aligned}
\phi_{1}(t) & v_{o}^{\frac{n+2}{n-2}}(t)+\phi_{2}(t) v_{\varepsilon}^{\frac{n+2}{n-2}}(t) \\
= & \phi_{1}(t) v_{o}^{\frac{n+2}{n-2}}(t)+\phi_{2}(t) v_{o}^{\frac{n+2}{n-2}}(t)+\phi_{2}(t)\left[v_{\varepsilon}^{\frac{n+2}{n-2}}(t)-v_{o}^{\frac{n+2}{n-2}}(t)\right] \\
= & {\left[\phi_{1}(t) v_{o}(t)+\phi_{2}(t) v_{o}(t)\right]^{\frac{n+2}{n-2}}+\phi_{2}(t)\left[v_{\varepsilon}^{\frac{n+2}{n-2}}(t)-v_{o}^{\frac{n+2}{n-2}}(t)\right] } \\
= & \left\{v(t)+\phi_{2}(t)\left[v_{o}(t)-v_{\varepsilon}(t)\right]\right\}^{\frac{n+2}{n-2}}+\phi_{2}(t)\left[v_{\varepsilon}^{\frac{n+2}{n-2}}(t)-v_{o}^{\frac{n+2}{n-2}}(t)\right]
\end{aligned}
$$

for $t \in[-\rho, \rho]$. We obtain

$$
\left|\left[-v^{\prime \prime}(t)+\frac{(n-2)^{2}}{4} v(t)\right]\left[\frac{n(n-2)}{4} v^{\frac{n+2}{n-2}}(t)\right]^{-1}-1\right|
$$




$$
\begin{aligned}
\leqslant & \left|\left\{1+\frac{\phi_{2}(t)}{v(t)}\left[v_{o}(t)-v_{\varepsilon}(t)\right]\right\}^{\frac{n+2}{n-2}}-1\right| \\
& +\frac{4}{n(n-2)} v^{-\frac{n+2}{n-2}}(t)\left\{\phi_{2}(t)\left|v_{\varepsilon}^{\frac{n+2}{n-2}}(t)-v_{o}^{\frac{n+2}{n-2}}(t)\right|\right. \\
& \left.+\left|\phi_{1}^{\prime}(t)\right|\left|v_{\varepsilon}^{\prime}(t)-v_{o}^{\prime}(t)\right|+\left|\phi_{1}^{\prime \prime}(t)\right|\left[v_{\varepsilon}(t)-v_{o}(t)\right]\right\}
\end{aligned}
$$

for $t \in[-\rho, \rho]$. It follows from Lemma 2.21, (2.16), (2.17), (3.1) and (3.4) that $v$ satisfies the equation

$$
v^{\prime \prime}-\frac{(n-2)^{2}}{4} v+\frac{n(n-2)}{4} K v^{\frac{n+2}{n-2}}=0 \quad \text { in } \mathbb{R},
$$

where $K$ is a smooth function on $\mathbb{R}$ such that

$$
|K(t)-1|=\left|\left[-v^{\prime \prime}(t)+\frac{(n-2)^{2}}{4} v(t)\right]\left[\frac{n(n-2)}{4} v^{\frac{n+2}{n-2}}(t)\right]^{-1}-1\right| \leqslant C_{1} \varepsilon^{2}
$$

for $t \in[-\rho, \rho]$, and $K \equiv 1$ in $\mathbb{R} \backslash[-\rho, \rho]$. Here $C_{1}$ is a positive constant that depends on $n$ only, so far as $\varepsilon>0$ is close to zero.

Let $\left\{\varepsilon_{i}\right\}$ be a decreasing sequence of small positive numbers such that $\lim _{i \rightarrow \infty} \varepsilon_{i}=0$. Denote the period of $v_{\varepsilon_{i}}$ by $T_{\varepsilon_{i}}$ for $i=1,2, \ldots$ With $\varepsilon_{1}$ small enough, we may assume that $T_{\varepsilon_{1}} \gg \rho$. We construct a positive smooth function by first gluing $v_{o}$ and $v_{\varepsilon_{1}}$ on $[-\rho, \rho]$ as described above and call the resulting positive smooth function $v_{1}$. Note that $v_{1}=v_{\varepsilon_{1}}$ in $\mathbb{R}^{+} \backslash(0, \rho)$. As $v_{\varepsilon_{1}}\left(t+T_{\varepsilon_{1}}\right)=v_{\varepsilon_{1}}(t)$ for $t \in \mathbb{R}$ and $v_{\varepsilon_{1}}$ and $v_{\varepsilon_{2}}$ are close to $v_{o}$ near $[-\rho, \rho]$, we let

$$
\tilde{v}_{\varepsilon_{2}}(t)=v_{\varepsilon_{2}}\left(t-T_{\varepsilon_{1}}\right) \quad \text { for } t \in \mathbb{R},
$$

and glue $\tilde{v}_{\varepsilon_{2}}$ and $v_{1}$ (that is, $v_{\varepsilon_{1}}$ ) on $\left[T_{\varepsilon_{1}}-\rho, T_{\varepsilon_{1}}+\rho\right]$ in a process similar to the one described above. Call the resulting function $v_{2}$. We continue to glue the solutions on the intervals

$$
\left[T_{\varepsilon_{1}}+T_{\varepsilon_{2}}-\rho, T_{\varepsilon_{1}}+T_{\varepsilon_{2}}+\rho\right], \ldots,\left[\sum_{k=1}^{i} T_{\varepsilon_{k}}-\rho, \sum_{k=1}^{i} T_{\varepsilon_{k}}+\rho\right], \ldots
$$

by $v_{\varepsilon_{3}}, \ldots, v_{\varepsilon_{i+1}}, \ldots$, respectively, after shifting appropriately. In particular, in the $(i+1)$ th step, let

$$
\tilde{v}_{\varepsilon_{i}}(t)=v_{\varepsilon_{i}}\left(t-\sum_{k=1}^{i-1} T_{\varepsilon_{k}}\right) \quad \text { and } \quad \tilde{v}_{\varepsilon_{i+1}}(t)=v_{\varepsilon_{i+1}}\left(t-\sum_{k=1}^{i} T_{\varepsilon_{k}}\right) \quad \text { for } t \in \mathbb{R},
$$

and glue $\tilde{v}_{\varepsilon_{i+1}}$ with $\tilde{v}_{\varepsilon_{i}}$ on the interval $\left[\sum_{k=1}^{i} T_{\varepsilon_{k}}-\rho, \sum_{k=1}^{i} T_{\varepsilon_{k}}+\rho\right]$. Finally we obtain a positive smooth function $v$ on $\mathbb{R}$ which satisfies the equation

$$
v^{\prime \prime}-\frac{(n-2)^{2}}{4} v+\frac{n(n-2)}{4} K v^{\frac{n+2}{n-2}}=0 \quad \text { in } \mathbb{R}
$$


for some smooth function $K$ such that

$$
|K(t)-1| \leqslant C_{2} \varepsilon_{1}^{2} \quad \text { for } t \in \mathbb{R},
$$

where $C_{2}$ is a positive constant depending on $n$ only. We may choose $\varepsilon_{1}>0$ as small as we like. We also have

$$
\begin{aligned}
& v\left(\sum_{k=1}^{i} T_{\varepsilon_{k}}-T_{\varepsilon_{i}} / 2\right)=v_{i}\left(T_{\varepsilon_{i}} / 2\right)=\varepsilon_{i} \rightarrow 0 \quad \text { and } \\
& v\left(\sum_{k=1}^{i} T_{\varepsilon_{k}}\right) \rightarrow 1^{-} \text {as } i \rightarrow \infty .
\end{aligned}
$$

As $v(t)=v_{o}(t)$ for $t \leqslant-\rho$, by (2.6) and (2.7), the corresponding solution $u$ related to $v$ by (2.1) is smooth across the origin. Thus $v$ corresponds to an exotic solution $u$ of Eq. (1.1) through (2.1).

Given a positive function $\varphi(r)$ defined for $r \gg 1$ which satisfies (1.12), let $\psi(t)=$ $\varphi\left(\mathrm{e}^{t}\right)$. It follows that $\psi$ is defined for $t \gg 1$ and

$$
\mathrm{e}^{(n-2) t / 2} \psi(t)
$$

is non-decreasing for $t \gg 1$ and unbounded from above. Let

$$
\varpi(t)=\ln \left[\mathrm{e}^{(n-2) t / 2} \psi(t)\right] \quad \text { for } t \gg 1 .
$$

We have $\lim _{t \rightarrow \infty} \varpi(t)=\infty$. Choose a decreasing sequence of numbers $\left\{\varepsilon_{i}\right\}$ such that $\varepsilon_{1}$ is small enough and the corresponding periods $T_{\varepsilon_{i}}$ of $v_{\varepsilon_{i}}$ satisfy the relation

$$
\varpi\left(T_{\varepsilon_{i}}\right) \geqslant \frac{n-2}{2} \sum_{k=1}^{i-1} T_{\varepsilon_{k}} \quad \text { for } i=2,3, \ldots
$$

By gluing the solutions $v_{o}, v_{\varepsilon_{i}}, i=1,2, \ldots$, as described above, we obtain a positive smooth function $v$ which satisfies Eq. (3.7) for a smooth function $K$. Suppose that

$$
t \notin[-\rho, \rho] \cup\left[T_{\varepsilon_{1}}-\rho, T_{\varepsilon_{1}}+\rho\right] \cup \cdots \cup\left[\sum_{k=1}^{i} T_{\varepsilon_{k}}-\rho, \sum_{k=1}^{i} T_{\varepsilon_{k}}+\rho\right] \cup \cdots,
$$

then $K(t)=1$. Suppose that

$$
t \in\left[\sum_{k=1}^{i} T_{\varepsilon_{k}}-\rho, \sum_{k=1}^{i} T_{\varepsilon_{k}}+\rho\right] \quad \text { for some } i \in \mathbb{N} .
$$

According to the construction above and Lemma 2.10, we have

$$
|K(t)-1| \leqslant C_{3} \varepsilon_{i}^{2} \leqslant C_{4} \exp \left(-\frac{n-2}{2} T_{\varepsilon_{i}}\right)
$$




$$
\begin{aligned}
& =C_{4} \exp \left(-\frac{n-2}{2} T_{\varepsilon_{i}}-\varpi(t)+\varpi(t)\right) \\
& \leqslant C_{3} \exp \left(-\frac{n-2}{2} \sum_{k=1}^{i} T_{\varepsilon_{k}}\right)\left[\mathrm{e}^{(n-2) t / 2} \psi(t)\right] \leqslant C_{4} \exp \left(\frac{n-2}{2} \rho\right) \psi(t),
\end{aligned}
$$

where $C_{3}$ and $C_{4}$ are positive constants that depend on $n$ only. Hence we obtain $|K(t)-1| \leqslant C_{5} \psi(t)$ for $t \gg 1$ and for a positive constant $C_{5}$. The corresponding solution $u$ is an exotic solution of Eq. (1.1) which satisfies (1.13). We note that $K(t)$ in this case is not monotonic for large $t$.

\section{REFERENCES}

[1] Bahri A., Coron J., The scalar-curvature problem on standard three-dimensional sphere, J. Func. Anal. 95 (1991) 106-172.

[2] Caffarelli L., Gidas B., Spruck J., Asymptotic symmetry and local behavior of semilinear elliptic equations with critical Sobolev growth, Comm. Pure Appl. Math. 42 (1989) 271297.

[3] Chang K.-C., Liu J.-Q., On Nirenberg's problem, Internat. J. Math. 4 (1993) 35-58.

[4] Chang S.-Y., Yang P., A perturbation result in prescribing scalar curvature on $S^{n}$, Duke Math. J. 64 (1991) 27-69.

[5] Chen C.-C., Lin C.-S., On compactness and completeness of conformal metrics in $\mathbf{R}^{N}$, Asian J. Math. 1 (1997) 549-559.

[6] Chen C.-C., Lin C.-S., Estimates of the conformal scalar curvature equation via the method of moving planes, Comm. Pure Appl. Math. 50 (1997) 971-1019.

[7] Chen C.-C., Lin C.-S., Estimates of the conformal scalar curvature equation via the method of moving planes. II, J. Differential Geom. 49 (1998) 115-178.

[8] Chen C.-C., Lin C.-S., On the asymptotic symmetry of singular solutions of the scalar curvature equations, Math. Ann. 313 (1999) 229-245.

[9] Chen W.-X., Li C.-M., A necessary and sufficient condition for the Nirenberg problem, Comm. Pure Appl. Math. 48 (1995) 657-667.

[10] Cheung K.-L., Leung M.-C., Asymptotic behavior of positive solutions of the equation $\Delta u+K u^{(n+2) /(n-2)}=0$ in $\mathbb{R}^{n}$ and positive scalar curvature, in: Discrete Contin. Dynam. Systems, Added Volume, Proceedings of the International Conference on Dynamical Systems and Differential Equations, 2001, pp. 109-120.

[11] Delaunay C., Sur la surface de revolution dont la courbure moyenne est constante, J. de Mathématiques 6 (1841) 309-320.

[12] Ding W.-Y., Ni W.-M., On the elliptic equation $\Delta u+K u^{(n+2) /(n-2)}=0$ and related topics, Duke Math. J. 52 (1985) 485-506.

[13] Fowler R., Further studies of Emden's and similar differential equations, Quart. J. Math. Oxford Ser. 2 (1931) 259-288.

[14] Gidas B., Ni W.-M., Nirenberg L., Symmetry and related properties via the maximum principle, Comm. Math. Phys. 68 (1979) 209-243.

[15] Gidas B., Ni W.-M., Nirenberg L., Symmetry of positive solutions of nonlinear elliptic equations in $\mathbb{R}^{n}$, in: Mathematical Analysis and Applications, Part A, Adv. in Math. Suppl. Stud., 7a, Academic Press, New York, 1981, pp. 369-402.

[16] Korevaar N., Mazzeo R., Pacard F., Schoen R., Refined asymptotics for constant scalar curvature metrics with isolated singularities, Invent. Math. 135 (1999) 233-272. 
[17] Leung M.-C., Conformal scalar curvature equations on complete manifolds, Comm. Partial Differential Equations 20 (1995) 367-417.

[18] Leung M.-C., Asymptotic behavior of positive solutions of the equation $\Delta_{g} u+K u^{p}=0$ in a complete Riemannian manifold and positive scalar curvature, Comm. Partial Differential Equations 24 (1999) 425-462.

[19] Leung M.-C., Growth estimates on positive solutions of the equation $\Delta u+K u^{(n+2) /(n-2)}=$ 0 in $\mathbb{R}^{n}$, Canad. Math. Bull., to appear.

[20] Li Y.-Y., Prescribing scalar curvature on $\mathbf{S}^{n}$ and related problems, part I, J. Differential Equations 120 (1995) 319-410.

[21] Li Y.-Y., Prescribing scalar curvature on $\mathbf{S}^{n}$ and related problems, part II: existence and compactness, Comm. Pure Appl. Math. 49 (1996) 541-597.

[22] Lin C.-S., Estimates of the conformal scalar curvature equation via the method of moving planes III, Comm. Pure Appl. Math. 53 (2000) 611-646.

[23] Loewner C., Nirenberg L., Partial differential equations invariant under conformal or projective transformations, in: Contributions to Analysis (a collection of papers dedicated to Lipman Bers), Academic Press, New York, 1974, pp. 245-272.

[24] Mazzeo R., Pacard F., Constant scalar curvature metrics with isolated singularities, Duke Math. J. 99 (1999) 353-418.

[25] Mazzeo R., Pollack D., Uhlenbeck K., Moduli spaces of singular Yamabe metrics, J. Amer. Math. Soc. 9 (1996) 303-344.

[26] Schoen R., The existence of weak solutions with prescribed singular behavior for a conformally invariant scalar equation, Comm. Pure Appl. Math. 41 (1988) 317-392.

[27] Taliaferro S., On the growth of superharmonic functions near an isolated singularity, I., J. Differential Equations 158 (1999) 28-47. 